\title{
Kirchliche Kommunikation im Wertewandel Gedanken zu einer neuen Religionspublizistik
}

von Winfried B. Lerg*

Es war einmal - in illo tempore - um eine Wendung zu gebrauchen, wie sie die ersten christlichen Publizisten bisweilen an den Anfang ihrer guten Nachrichten, ihrer Frohen Botschaften, gestellt haben, - in jener Zeit, als der Niederländer Henk Prakke Professor für Publizistik in Münster war, kamen nacheinander zwei neue Assistenten an sein Institut: Der eine war Redakteur einer evangelischen Jugendzeitschrift, der andere Redakteur einer katholischen Bistumszeitschrift. Der erste hieß Gerhard Stoll, der andere Michael Schmolke. Beide schrieben sie an ihrer Dissertation. Henk Prakke, Buchverleger aus Assen und Dozent für Publizistik an der Universität Groningen, Mitglied in der freisinnig-protestantischen Rundfunkvereinigung seines Landes, hatte, als er nach Münster berufen worden war, wenige Jahre zuvor (1957) sein fachwissenschaftliches Standardwerk veröffentlicht unter dem Titel: „De Samenspraak in onze Samenleving (Das Gespräch in unserem Zusammenleben) ${ }^{4}{ }^{1}$; später prägte er daraus den Titel seiner Münsteraner Buchreihe: „Dialog der Gesellschaft“.

In seiner Groninger Buchreihe war eine kleine Studie erschienen, die, wie in einem Punktlicht, ein besonderes wissenschaftliches Interesse Henk Prakkes erkennen ließ. Der Titel der Arbeit lautete „Kerkgang om Nieuws (etwa Kirchenbesuch, um Neues zu erfahren) ${ }^{\star} .{ }^{3}$ Beschrieben und gedeutet wurde, kurz gesagt, der in vielen niederländischen Gemeinden bis in die Neuzeit anzutreffende Brauch, daß die Gläubigen bei ihrem sonntäglichen Kirchgang sich vor zwei verschiedenen Kanzeln versammelten: In der Kirche, wo sie das Evangelium, die Gottesnachricht, hörten, und anschließend vor der Kirche, vor einer besonderen Kanzel, wo ihnen der Pfarrer die Lokalnachrichten, und nicht nur die Gemeindeverkündigungen, mitteilte.

Die pastorale Bipolarität, wie sie in jener kirchen- und kommunikationsgeschichtlichen Figur aufscheint, - die Kanzel in der Kirche und die Kanzel vor der Kirche - sollte bald darauf das theoretische Grundmuster abgeben für den von Prakke und Stoll im Jahre 1968 veröffentlichten Aufsatz mit dem Titel „Entwurf zu einer Religionspublizistik $^{\star 4}$; Gerhard Stoll nahm übrigens zu jener Zeit bereits im 12. Semester einen Lehrauftrag für Religiongspublizistik am Institut für Publizistik in Münster wahr. Ich werde gleich noch einmal auf diesen Programmaufsatz von Prakke und Stoll zurückkommen.

Mein „snelldialoog“, wie Prakke den kurzen Blick unserer Umweltwahrnehmung umschrieben hat, wäre unvollständig, ohne die Dissertation jener beiden Assistenten, Stoll und Schmolke, zu erwähnen. Dazu soll daran erinnert sein, daß sie ihre Dissertationsthemen noch von Prakkes Vorgänger, von Walter Hagemann, bekommen hatten. Hagemann, letzter Chefredakteur der Berliner Zentrums-Zeitung „Germania“ bis zum Verbot des Blattes durch die nationalsozialistische Reichspressekammer im Jahre 1938 und als Münsteraner Professor für Publizistik nach dem Krieg - neben seinem Berliner Kollegen Emil Dovifat - in der Gesellschaft katholischer Publizisten und in der CDU

* Prof. Dr. Winfried B. Lerg ist Direktor des Instituts für Publizistik der Westfälischen Wilhelms-Universität Münster. - Der Text ist eine Geburtstagsrede, nicht für ${ }_{n}$ Communicatio Socialis", wie man auch glauben könnte, sondern für Dr. Gerhard E. Stoll, den geschäftsführenden Direktor des Evangelischen Presseverbandes Westfalen und Lippe e.V. Stoll, einer der Paten von CS, der am 16. März 198960 Jahre alt wurde. 
tätig, hatte den Lehr- und Forschungsschwerpunkt „Publizistik im Dritten Reich“ entwickelt und bis zu seinem Ausscheiden ein rundes Dutzend Dissertationen aus diesem Themenbereich betreut.

Gerhard Stoll schrieb seine akademische Abschlußarbeit über kirchliche Kommunikationsgeschichte im ersten Jahr der nationalsozialistischen Herrschaft zum Thema „Die Evangelische Zeitschriftenpresse im Jahre 1933“. ${ }^{5}$ Es ging ihm bei seiner Untersuchung um das Schicksal der Herausgeber, Redakteure, Mitarbeiterinnen und Mitarbeiter jener Zeitschriften, und er schilderte das Ende der vielfältigen und einflußreichen „Evangelischen Publizistik“, - ein Begriff übrigens, den August Hinderer geprägt hatte. ${ }^{6}$

Michael Schmolke schrieb als Dissertation eine bis heute nicht überholte publizistische Biographie von Adolf Kolping, des rheinischen Gesellenvaters, der von der katholischen Sozial- und Kommunikationsgeschichte seither nicht mehr übersehen wird.' Nun werden Sie sagen: Das waren geschichtliche Arbeiten. Doch die ereignisbezogene, historisch-kritische Methode war zu jener Zeit in der Publizistikwissenschaft, und nicht nur in diesem Fach, das erkenntnistheoretisch besterprobte Untersuchungs- und Erklärungsverfahren. Gleichwohl waren zugleich Spurenelemente auszumachen, die auf eine strukturbezogene, sozialwissenschaftliche Methode hinwiesen. Die alte Zeitungswissenschaft, sofern sie nicht eine einfache Medienkunde war, operierte mit der Struktur „privat-öffentlich" und suchte nach der „öffentlichen Meinung " in Geschichte und Gegenwart. Hagemann führte das dogmengeschichtlich entscheidende Schema vom publizistischen Prozeß ein: Publizist - Aussage - Publikum. Es wurde von Henk Prakke, wie er immer zu sagen pflegte, ,erweitert und verfeinert “ und der internationalen Fachsprache angepaßt: Kommunikator - Medium - Rezipient. Der publizistische Prozeß Hagemanns stand für das gesellschaftliche Zwiegespräch, für den Dialog der Gesellschaft Prakkes. Nun war es möglich, das publizistische Geschehen im gesellschaftlichen Kontext verstehend oder analytisch darzustellen und zu deuten, seine Strukturen und Funktionen, seine Ursachen und Folgen besser zu erkennen. Studierende beider theologischer Fakultäten besuchten seiner Zeit die Seminare von Prakke, seines Assistenten Schmolke und besonders seines Lehrbeauftragten für Religionspublizistik, Gerhard Stoll.

Noch in der Aufbruchsstimmung des Zweiten Vatikanischen Konzils hatten Schmolke und der Prakke-Schüler Pater Franz Josef Eilers eine wichtige Fachzeitschrift gegründet, unter dem an das berühmte Dekret erinnernden Titel: "Communicatio Socialis“, Untertitel: „Zeitschrift für Publizistik in Kirche und Welt". Im ersten Jahrgang 1968 dieser Zeitschrift erschien der erwähnte programmatische Aufsatz von Prakke und Stoll, jener "Entwurf zur Religionspublizistik“. Der Beitrag ging weit hinaus über das, was bisher in der Wissenschaft und in der fachjournalistischen Essayistik über Kirchenpublizisten, Kirchenmedien und über die Praxis eines christlichen Journalismus und seiner Verbände in beiden Kofessionen vorgetragen worden war.

Erlauben Sie mir bitte ein kurzes Zitat aus diesem Aufsatz: „Die Erforschung und Beschreibung der religiösen Determinante im publizistischen Geschehen hätte sich die 'Religionspublizistik' zur Aufgabe zu stellen. Sie will die öffentliche Kommunikation zeitbezogener, religiöser, konfessioneller, bekenntnisgebundener Erfahrung in der Gesellschaft erforschen. Die 'Erfahrung des Heiligen' und deren Wirkung auf das gesellschaftliche $Z$ wiegespräch manifestiert sich wechselseitig bezogen in der religiösen Bedingtheit religiöser Aussage. ${ }^{8}$

Das Bild von den zwei getrennten Kanzeln ist hier verblaßt. An seine Stelle setzen Prakke und Stoll ihr Konzept einer christlichen Kommunikationsgesellschaft, und diese Gesellschaft ist ein offenes Forum, ja, auch ein Marktplatz. Verkündigung bleibt kein 
theologisches Theorem, sie ist, um Walter Hagemanns klassisches Stenogramm anzuführen, „die öffentliche Aussage aktueller Bewußtseinsinhalte ${ }^{\text {“ }}{ }^{9}$ Verkündigung ist unser aller existenzielles kommunikatives Handeln als Christen.

Müßig geworden ist darum die nachgerade scholastische Verortung der Kanzel, das eine Mal in der Kirche und das andere Mal vor der Kirche; ich meine damit nicht allein das Gotteshaus, sondern eher noch die Organisation, das kirchliche Amt und seine Behörden. Die jüngste kirchliche Kommunikationsgeschichte ist eine Geschichte des reaktiven Handelns: Sobald draußen auf dem Kirchplatz eine neue Kanzel errichtet worden war, ist sie erst einmal mißtrauisch beobachtet, bisweilen sogar empört bekämpft worden. Schließlich stellte man trotzig seine eigene Kanzel gleich daneben auf: Gegenpublizistik. Als die moderne Presse entstanden war, folgte alsbald die kirchliche, die gern sogenannte „konfessionelle“ Presse, mitsamt den evangelischen Presseverbänden und mit dem Augustinus-Verein. Als der Film aufkam, sollte es, nach anhaltenden religionspädagogischen und moraltheologischen Auseinandersetzungen, auch einen kirchlichen Film geben.

Kommunikationssystematisch gesprochen, handelte es sich um Supplementierung, um zusätzliche Medien, die von den Kirchen in die neue Medienwelt gesetzt worden sind.

Beim Rundfunk, zunächst beim Radio, später auch beim Fernsehen, ging es nur um Nuancen anders zu, - in den meisten europäischen Ländern übrigens. Eine eigene Kanzel, der berühmte Kirchensender, war vom staatlichen Träger der Fernmeldehoheit nicht zu bekommen. Deshalb wurde auf administrativem Wege ein bescheidener Programmplatz für die kirchliche Rundfunkkanzel erstritten, für die „Morgenandacht“ oder „Das Wort zum Sonntag“. Eine komplementäre, ergänzende Lösung, heißt das in der Kommunikationssystematik.

Doch die Lehre von den zwei Kanzeln, wie ich sie einmal nennen möchte, blieb unbestritten. Erinnern Sie sich an den Untertitel der Zeitschrift "Communicatio Socialis“? Er lautet: „Zeitschrift für Publizistik in Kirche und Welt“. Das Wort „und" verbindet noch nicht, sondern es stellt zunächst einmal nur zusammen, es koordiniert, wie in den Ausdruckspaaren „Kapital und Arbeit“, „Individuum und Gesellschaft“, „Geist und Seele", oder wie in dem Titel "Christ und Welt". Wo immer solche koordinierten Ausdruckspaare auftreten, haben wir es mit einem Vermittlungsproblem zu tun, mit einem latenten Kommunikationsbedarf. Und er wird, wenn überhaupt, nur unzureichend gedeckt durch supplementäre oder komplementäre Strategien, durch eine Kirchenkanzel und eine Weltkanzel, durch kirchliche Medien auf der einen und weltliche Medien auf der anderen Seite des publizistischen Spektrums einer Gesellschaft. Das kann zwar nicht bedeuten, daß Kirchenmedien überflüssig seien oder wirkungslos, aber - erlauben Sie mir das Bild - sie leben gefährlich, die Kirchenmedien. In einer offenen Gesellschaft können Differenzen mit Richtlinienkompetenzen der herausgebenden Kirchenbehörde oder deren Finanzabteilung, nicht zuletzt wegen zurückgehender Reichweiten, auftreten. In geschlossenen Gesellschaften werden Kirchenmedien gewöhnlich dezimiert, ihre Art und Anzahl staatlich vorgeschrieben (Gerhard Stoll hat das für Deutschland im Jahre 1933 untersucht), oder sie werden zensiert oder verboten (wir haben es kürzlich erst wieder aus der DDR gehört).

Die strukturelle Labilität der Kirchenmedien macht nach meiner Beobachtung das entscheidende Moment bei dem genannten Vermittlungsproblem in Kirche und Welt aus, vor dem manche Kirchenpublizisten mit ihrem Publikum bisweilen ihre Ratlosigkeit erkennen müssen. Kircheneigene Medien laufen in offenen Kommunikationsgesellschaften, die - zugleich ideologisch wie ökonomisch gesehen - publizistische Wettbewerbsgesellschaften darstellen, ständig Gefahr, isoliert zu werden. Allzuoft müssen sie 
ihren geistigen und geistlichen Mitbestimmungsanspruch im gesellschaftlichen Wertewandel preisgeben. Nicht wenige verlieren ihre Aufgabe aus den Augen, ohne, mit den Worten von Prakke und Stoll, ,die Grundströmungen der geistigen Kräfte auszuloten, die das öffentliche Bewußtsein in der Gesellschaft mit Hilfe publizistischen Geschehens mitbestimmen". ${ }^{10}$

Nach meiner Auffassung ist nicht die Vermittlung von Kirche und Welt das erste publizistische Gebot - hier mögen Maßnahmen und Medien der Öffentlichkeitsarbeit, der Public Relations, zureichen. Das religionspublizistische Postulat - und nun lasse ich mich von meinem Lehrer Henk Prakke, er feierte im April 1989 seinen 90. Geburtstag, und von meinem Freund Gerhard Stoll, dessen 60. Geburtstag wir am 16.3.1989 feierten, an die Hand nehmen - das religionspublizistische Postulat also, sieht die Vermittlungsfigur als ein Prinzip christlichen Kommunikationshandelns.

Im gesellschaftlichen Spannungsfeld wertebestimmender Verkündigung an einem Pol und werterealisierender Publizistik am anderen Pol teilen wir uns mit, indem wir miteinander austauschen. In der Wertekommunikation der Christen müssen die spitzköpfigen Vordenker wie die rundköpfigen Nachdenker keineswegs zuerst und zu lange den Kompaß in der Hand haben, um damit die Richtung zu bestimmen, die der Wertewandel nehmen soll. Wir alle sind aufgerufen, die Agenda für den christlichen Dialog der Gesellschaft gemeinsam zu entwerfen.

Den Medien wird nachgesagt, - die Kommunikationswissenschaft hat es untersucht und bestätigen müssen - daß sie sich ihre Themen selbst machen, anstatt sie bei den Menschen aufzusuchen, bei ihrem Publikum, dessen publizistischer Anwalt sie einer verbreiteten journalistischen Berufsideologie nach sein sollten.

Mit diesem letzten Hinweis geht es mir jedoch weder um die Thematisierungshypothese der Kommunikationswissenschaft noch um eine journalistische Berufsideologie. Vielmehr möchte ich damit die Aufmerksamkeit zum Schluß auf die kritische Qualität der Religionspublizistik lenken. Wenn Prakke und Stoll behaupten, die publizistische Manifestation des Religiösen sei eine Frage der öffentlichen Intention, Darstellung und Wirkung letzter gesellschaftsprägender Axiome, religiöse Communio werde zur öffentlichen Kommunikation, dann ist dem uneingeschränkt zuzustimmen.

Ich darf, um mit Henk Prakke zu sprechen, ein wenig ,erweitern und verfeinern“ und behaupten, die publizistische Manifestation des Religiösen ist eine Frage der Wertebestimmung, der Wertedarstellung und der Werteverwirklichung im Dialog der Gesellschaft. Damit begreifen wir die Dynamik des Wertewandels und die kommunikative Verpflichtung, die uns durch die Verkündigung daraus erwächst.

\section{Anmerkungen}

1 H.(endricus) J.(ohannes) Prakke: De samenspraak in onze samenleving. Inleiding tot de publicistiek, Assen 1957

2 Die Buchreihe: „Dialog der Gesellschaft “ wurde eröffnet mit der von Henk Prakke, Winfried B. Lerg und Michael Schmolke überarbeiteten und ergänzten 2. Auflage von Walter Hagemann: Grundzüge der Publizistik, Münster 1966 (zuerst 1947)

3 H.J. Prakke: Kerkgang om nieuws. De Kerkespraak, Assen 1955 (= Publicistische Publicaties No.2)

4 Henk Prakke und Gerhard E. Stoll: Entwurf zu einer Religionspublizistik, in: Communicatio Socialis, 1.Jg., 1968, H.1, S. 34-37

5 Gerhard E. Stoll: Die evangelische Zeitschriftenpresse im Jahre 1933, Witten 1963, zugleich Universität Münster, Phil. Diss. v. 26.07.1962

6 August Hinderer (1877-1945) war seit 1918 geschäftsführender Vorsitzender und Direktor des Evangelischen Preßverbandes für Deutschland, seit 1925 Lehrbeauftragter für evangeli- 
sches Pressewesen, seit 1927 Honorarprofessor, seit 1931 Direktor des von ihm gegründeten Seminars für Publizistik der Evangelisch-Theologischen Fakultät der Universität Berlin. Vgl. auch Walter Schwarz: August Hinderer. Leben und Werk, Stuttgart 1951

7 Michael Schmolke: Adolph Kolping als Publizist, Münster 1966 (= Dialog der Gesellschaft, Bd. 3) zugleich Universität Münster, Phil.Diss.v. 16.01.1965

8 Henk Prakke; Gerhard E. Stoll: Entwurf zu einer Religionspublizistik, a.a.O., S.35

9 Walter Hagemann: Grundzüge der Publizistik, a.a.O., S.37

10 Henk Prakke; Gerhard E. Stoll, a.a.O.

\section{Summary}

Lerg refers in his work to an article by Henk Prakke and Gerhard Stoll dealing with basic aspects of church media and communications which appeared in "Communicatio Socialis" in its first year of publication in 1968. In the article entitled „Towards a Religious Journalism“ Prakke and Stoll posit a Christian communications society that is open to new forms of communication. As opposed to this, the recent history of chruch communications can be seen, according to Lerg, as a history of reactions. The Church reacts to developments in the media by counter publications: the church media are to be found opposite the secular media at the other end of the communications spectrum of a society. Now, as a result of their particular structures church media are in danger of becoming isolated in open communications societies which, both ideologically and financially, are medially competitive societies. Nevertheless, as Prakke and Stoll hold, precisely the function of transferring information etc. can be seen as a vital principle of chruch communications. Thus Lerg concludes that the manifestation of the religios through the media is ultimately a question of presenting and realizing values in a dialogue with society.

\section{Résumé}

Dans son exposé, Lerg se réfère à un article de Henk Prakke et de Gerhard Stoll sur les aspects fondamentaux du journalisme religieux, paru dans la première année de la "Communicatio Socialis“ (1968). Prakke/Stoll élaborent, dans leur "Esquisse du journalisme religieux “ (titre), le brouillon d'une société chrétienne de communication qui est sans réserve à l'egard de nouvelles formes de communication. Par contre, la plus récente histoire de la communication ecclésiastique peut étre considé rée - selon Lerg - comme une histoire d'action "réactive". On a répondu au développement de nouveaux média par un „contre-journalisme“ ecclésiastique: Dans l'éventail journalistique de la société, les média laics ont été mis en regard des média ecclésiastiques risquent d'étre isolés dans les sociétés de communication ouvertes, qui sont - sur le plan idéologique et économique - des sociétés compétitives médiatisées. Or, la fonction de médiation peut, selon Prakke/Stoll, ètre regardée comme un principe important de l'action communicative chrétienne. Lerg affirme donc qu'une manifestation de la religion dans les mass-média implique, au bout du compte, la présentation et la réalisation des valeurs dans le dialogue de la société.

\section{Resumen}

En sus explicaciones, Lerg se refire a un articulo aparecido en el primer ano de publicacion de "Communcatio Socialis“, en 1968, de Henk Prakke y Gerhard Stoll sobre aspectos fundamentales del peiodismo eclesiástico. Prakke/Stoll elaboran en su "modelo para periodismo religioso" el título de su articulo - el concepto de una sociedad de communicación christiana - que está abierta a nuevas formas de communicaión. La resciente historia de communicación eclesiástica se puede - según muestra Lerg - considerar como historia de procedimiento reactivo. Al desarrollo de nuevos medios se responde con contra-publicaciones eclesiásticas: a los medios profanos, al otro lado del espectro del periodismo de una sociedad se opusieron medios eclesiásticos. Por consecuencia de sus estructuras especificas, los medios eclesiásticos en sociedades de comunicación abiertas, que - ideológica y economicamente - son entidades de publicidad para periodismo, corren peligro de ser aislados. Sin embargo es especialmente la funcion de mediación, la que puede ser considerada como un principio importante en el procedimiento de comunicación cristiana, según Prakke/Stoll. Conforme a ésto, Lerg comprueba que la manifestación periodistica de lo religioso, es al fin y al cabo una cuestión de presentación y realización de valores en el diálogo con la sociedad. 